\title{
A 3D-printed stage adapter enabling non-destructive live imaging of Pachyclavularia violacea coral
}

Paul Wollerman ${ }^{1}$, Pauline Liu ${ }^{1}$, Abigail Saks ${ }^{1}$, Justin Seventko ${ }^{1}$, Clare Kennedy ${ }^{1}$ and David Reeves ${ }^{2}$

${ }^{1}$ Bergen County Academies, United States, ${ }^{2}$ Bergen County Academies, Hackensack, New Jersey, United States

Coral reefs are home to about $25 \%$ of marine species, but are rapidly disappearing due to recent massive coral bleaching events(1). Current coral imaging methods often stress or kill the animal and/or symbiotes. Observable changes in coral responses, particularly those related to stress and coral bleaching should reflect experimental manipulations rather than artefact. Observations and quantifications of polyp tentacle movement and algal properties may ideally be completed multiple times during the course of the experiment to monitor changes over days or weeks. A novel stage adapter (Confocal Coral Adapter, CCA) was designed and then 3D printed in polylactic acid (PLA) using a commercially available 3D fuseddeposition modelling printer (Ultimaker 5). The 3D print measures $50 \mathrm{~mm}$ long by $60 \mathrm{~mm}$ wide by 25 $\mathrm{mm}$ tall. The assembly also included two captive bolts for adjustment. Two interlocking arms suspend the coral fragment inverted above the confocal microscope in a thin glass-bottom confocal imaging dish filled with marine tank (salt) water (Figure 1). The height of the subject can easily be adjusted relative to the rest of the device. This permits high resolution confocal imaging and minimization of beam damage by allowing lower intensity illumination. Tentacle extensions during and after imaging, as well as a restoration of physiological functions when specimens were returned to the maintenance tank, showed that live imaging over time is possible using this method. The CCA device is adjustable for a small but useful range of coral fragment and plug sizes/heights. The adapter was used to study the effect of the bacterium Pseudomonas stutzeri on the health of Pachyclavularia violacea. The adapter enabled data collection in both confocal and regular epifluorescence microscopy. Fluorescence time series of moving polyp tentacles and micrographs of the corals' surface were made that revealed qualitative information about the effects of $P$. stutzeri on coral health, and allowed algae on the surfaces of certain coral to be identified as filamentous. Micrographs also offered the exact zooxanthellae count of a specific area, which may be an important indicator of the extent of coral bleaching. 


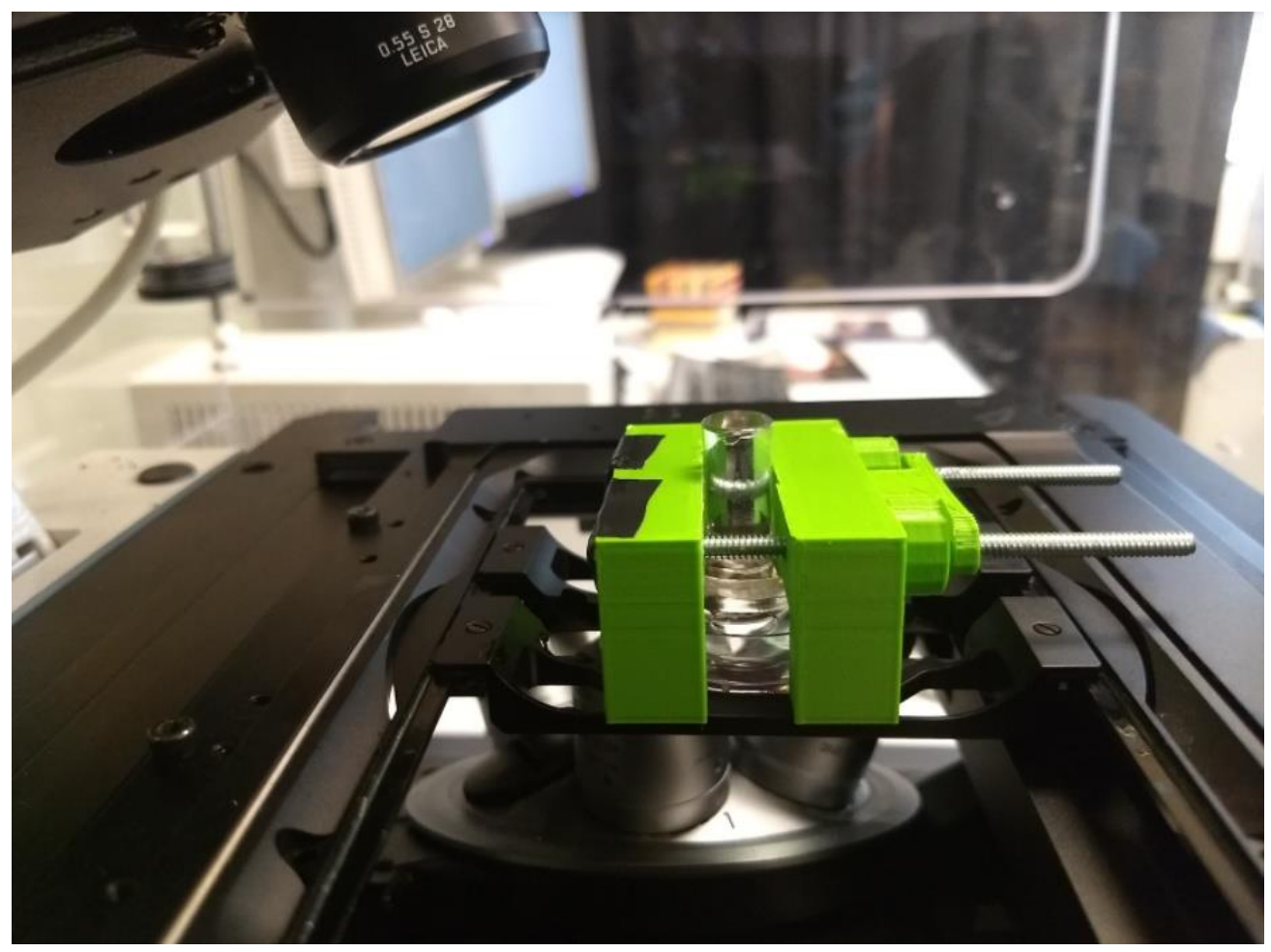

Figure 1. Confocal Coral Adapter in use for confocal microscopy (Leica TCS-SP5). Note the minimal distance to the objective lens made possible using this design.

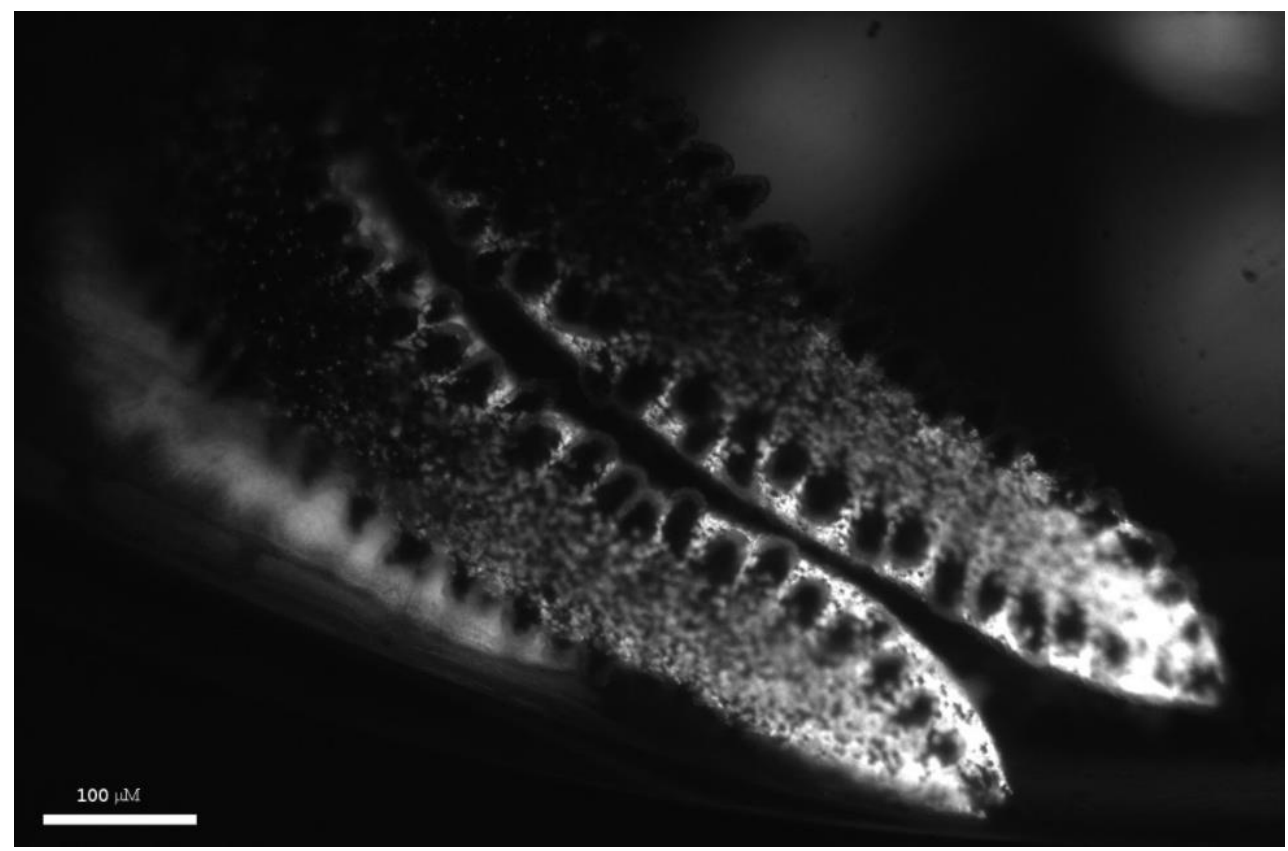

Figure 2. Live epifluorescence micrograph of Pachyclavularia violacea mounted using the CCA stage adapter. GFP cube, 4x objective lens.

\section{References}

(1) Putnam HM, Barott KL, Ainsworth TD, Gates RD. The Vulnerability and Resilience of Reef-Building Corals. Curr Biol. 2017 Jun 5;27(11):R528-R540. doi: 10.1016/j.cub.2017.04.047 